\title{
tic\&société
}

Vol. 10, $N^{\circ} 1 \mid 1$ er semestre 2016

Contrôle social, surveillance et dispositifs numériques

\section{Dossier commun des revues tic\&société et Terminal}

\section{Dominique Carré et Jacques Vétois}

\section{Q OpenEdition}

\section{Journals}

Édition électronique

URL : http://journals.openedition.org/ticetsociete/1970

DOI : 10.4000/ticetsociete. 1970

Éditeur

Association ARTIC

Référence électronique

Dominique Carré et Jacques Vétois, « Dossier commun des revues tic\&société et Terminal », tic\&société [En ligne], Vol. 10, № 1 | 1er semestre 2016, mis en ligne le 15 octobre 2016, consulté le 20 avril 2019. URL : http://journals.openedition.org/ticetsociete/1970 ; DOI : 10.4000/ticetsociete.1970

Ce document a été généré automatiquement le 20 avril 2019.

Licence Creative Commons 


\title{
Dossier commun des revues tic\&société et Terminal
}

\author{
Dominique Carré et Jacques Vétois
}

1 Avec l'appel à contributions de ce dossier commun "Contrôle social, surveillance et dispositifs numériques » des revues tic\&société et Terminal, nous souhaitions revenir sur la question du contrôle social, « que celui-ci soit mis en œuvre par les États, les entreprises ou les particuliers ». Signalons que nos deux revues sont déjà intervenues à plusieurs reprises sur l'analyse de la société à travers ce prisme.

2 Le renforcement du contrôle étatique sur les réseaux informatiques et téléphoniques justifié par les menaces terroristes et la situation internationale, d'une part, et celui mis en œuvre par les entreprises à la fois vis-à-vis de leurs salariés et de leur clientèle qu'il faut fidéliser, d'autre part, nécessitent de reprendre et d'approfondir l'analyse. Cette sollicitation est d'autant plus nécessaire et importante qu'avec le développement des réseaux sociaux numériques, les particuliers deviennent aussi acteurs en exportant des images de «soi » et produisent des traces exploitables à leur insu (et exploitées) à la fois par les services étatiques dans leurs stratégies de régulation et de contrôle, par les entreprises dans leur recherche de nouvelles segmentations des marchés, mais aussi dorénavant par les particuliers eux-mêmes. Les articles de ce dossier recoupent largement sur des terrains d'étude particuliers ces trois aspects.

3 Sous prétexte de lutter contre la cybercriminalité, plusieurs pays africains tels que l'Angola, le Gabon, le Kenya, le Cameroun, le Niger et la Côte d'Ivoire ont procédé par des moyens divers à l'identification des abonnés aux services d'Internet et de téléphonie mobile. Jean-Jacques Maomra Bogui et N'Guessan Julien Atchoua ont étudié le cas de la Côte d'Ivoire à l'issue d'une période de guerre civile dans les années 2010-2011 entre les partisans de Laurent Gbagbo et d'Alassane Ouattara. Cette identification des internautes est confiée à des entreprises qui sont des succursales des multinationales de l'informatique. Dans un pays déjà traumatisé par la guerre civile, cette surveillance de l'usage des TIC (et la répression qui ne manquera pas de s'ensuivre) conduit les usagers à s'interroger sur la préservation de la vie privée et des libertés. 
4 L'article d'Anne-France Kogan aborde le rôle des TIC dans l'évolution du métier des transporteurs routiers. À la fois comme facteur d'autonomie et de liaison avec leur famille lors des déplacements, mais aussi comme moyen de contrôle des horaires et des itinéraires par le service exploitation du maître d'œuvre. L'indépendance du chauffeur routier, l'une de leur fierté autrefois, est grignotée progressivement par la flexibilité que demande la hiérarchie de l'entreprise. Le respect des délais fixés (souvent par des algorithmes et des calculs sans tenir compte des réalités) et la surveillance a posteriori des traces laissées dans les divers dispositifs, dont des capteurs électroniques avec lesquels sont équipés les camions, imposent des contraintes de plus en plus fortes sur le personnel qui voit ses marges d'autonomie se réduire progressivement. Selon l'auteure, on assiste à la mise en œuvre graduelle d'une « société disciplinaire » et d'une « société de contrôle » dans le secteur des transports.

5 Johann Chaulet et de Camille Allaria ont enquêté tout à la fois dans un centre d'appels téléphoniques et dans un pôle de surveillance électronique des condamnés portant un bracelet électronique. Ces deux terrains d'étude sont intéressants et originaux. Dans le centre d'appels téléphoniques, les téléacteurs essaient de vendre des produits d'assurance et toutes leurs communications et transactions sont filtrées par un système informatique qui enregistre des données individualisées, comme le nombre de communications de chaque agent, leur durée, les succès et les échecs obtenus, etc. Des superviseurs contrôlent ainsi les groupes de téléacteurs. L'enquête du pôle de surveillance a été menée quant à elle au centre pénitentiaire des Baumettes, à Marseille. Des entretiens ont été menés avec les surveillants du pôle centralisateur et les appels téléphoniques aux porteurs de bracelet électronique ont été enregistrés avec leur accord. Les auteurs se sont intéressés aux techniques développées par les surveillés afin d'« endormir » la méfiance des surveillants dans les deux enquêtes. Dans chaque cas, il s'agit d'analyser les contournements du système soit sur le plan technique, tant il est vrai que chaque système de surveillance possède des failles, soit sur le plan de la négociation surveillant/surveillé en faisant appel à la compassion. Ainsi, les conditions de la surveillance peuvent être critiquées, voire améliorées pour les surveillés. Mais les principes sur lesquels elle s'appuie et la finalité du dispositif technique de surveillance ne sont pas remis en cause et ne peuvent pas l'être.

6 L'article de Mary Jane Kwok Choon traite de la participation d'une vingtaine d'étudiant (e)s du Québec au réseau social Facebook et les usages qu'ils ou elles en font. Il existe peu d'études qualitatives sur les usages de Facebook par de jeunes adultes qui l'utilisent à la fois pour leur travail universitaire et leurs loisirs. Par l'intermédiaire du « fil d'actualité », ils peuvent quotidiennement suivre les activités de leurs ami(e)s et de leurs réactions par rapport aux informations locales et nationales (posts d'articles, de photos, d'invitations, etc.). Bien entendu, cela implique réciproquement que chaque participant(e) à un réseau d'ami(e)s doit également se mettre en scène et fournir une image de soi qui s'intègre à l'ensemble. Progressivement, Facebook prend une place grandissante dans leur vie quotidienne et, après réflexion, certains utilisateurs tentent de se déconnecter du réseau et de se débarrasser des sollicitations trop pressantes. L'expérience semble prouver que la peur de l'exclusion de certaines activités sociales, de «manquer quelque chose», reste très forte et qu'en général la déconnexion est de courte durée. Néanmoins émerge chez les utilisateurs une réflexion critique sur leur pratique du réseau social numérique qui les pousse après la reconnexion à paramétrer leur site pour protéger, si cela est possible, leur vie privée. 
7 Pour compléter ce dossier, nous publions la contribution de Simon Borel, qui interroge la nature du pouvoir et la forme prise par celui-ci dans la "société en réseaux ». Ce qu'il appelle le "panoptisme horizontal » ou le "panoptique inversé » serait, selon lui, en émergence à l'intersection des médias sociaux numériques, du capitalisme cognitif, de la gouvernementalité néolibérale et d'une certaine dynamique démocratique contemporaine. Ce panoptique est à la fois à l'inverse du panoptique totalitaire, tout en se rapprochant en certains points de ce dernier. L'intérêt de cet article est de formaliser la manière dont la surveillance et le contrôle se caractérisent en ce début de $\mathrm{XXI}^{\mathrm{e}}$ siècle : surveillance de tous par tous en ligne, renouveau procédural et bureaucratique via Internet et nouvelles formes de contrôle marchand des flux désirants sur les réseaux.

Les coordinateurs de ce dossier conjoint, Dominique Carré pour tic\&société et Jacques Vétois pour Terminal, enfin, proposent, en ouverture de ce numéro, de présenter à grands traits, en empruntant une approche sociohistorique, l'évolution du contrôle social par les techniques informatiques que l'on appellerait plus volontiers aujourd'hui le numérique.

\section{AUTEURS}

DOMINIQUE CARRÉ

coéditeur de tic\&société, ticetsociete@openedition.org

\section{JACQUES VÉTOIS}

membre du comité de rédaction de Terminal, redaction@revue-terminal.org 